\title{
Tissue factor pathway inhibitor 2 as a serum marker for diagnosing asymptomatic venous thromboembolism in patients with epithelial ovarian cancer and positive D-dimer results
}

\author{
RYUTA MIYAKE $^{1}$, YUKI YAMADA ${ }^{1}$, SHOICHIRO YAMANAKA ${ }^{1}$, RYUJI KAWAGUCHI $^{1}$, \\ NORIHISA OOTAKE ${ }^{2}$, SHOHEI MYOBA ${ }^{2}$ and HIROSHI KOBAYASHI ${ }^{1,3}$ \\ ${ }^{1}$ Department of Obstetrics and Gynecology, Nara Medical University, Kashihara, Nara 634-8522; \\ ${ }^{2}$ Bioscience Division, Research and Development Department, Tosoh Corporation, Ayase-shi, Kanagawa 252-1123; \\ ${ }^{3}$ Department of Gynecology, Ms.Clinic MayOne, Kashihara, Nara 634-0813, Japan
}

Received November 11, 2021; Accepted December 14, 2021

DOI: $10.3892 / \mathrm{mco} .2021 .2479$

\begin{abstract}
Tissue factor pathway inhibitor 2 (TFPI2) is a serodiagnostic marker for epithelial ovarian cancer (EOC) and is the primary inhibitor of the extrinsic coagulation pathway. The present study assessed the diagnostic performance of TFPI2 for detecting venous thromboembolism (VTE) in patients with EOC and positive D-dimer results $(>1.0 \mu \mathrm{g} / \mathrm{ml})$. First, the clinical data of 81 patients with EOC admitted to Nara Medical University Hospital between January 2008 and December 2015 were collected. Also, 25 patients with VTE and 56 patients without VTE were included. Receiver-operating characteristic (ROC) curve analyses were performed to determine the diagnostic efficacy of TFPI2 in discriminating patients with VTE from those without VTE. Serum TFPI2 levels in patients with VTE were significantly higher than in non-VTE patients (median, 472.2 vs. $279.1 \mathrm{pg} / \mathrm{ml}, \mathrm{P}<0.001$ ). Using the Youden index, the optimal cutoff value for the TFPI2 level was set at $398.9 \mathrm{pg} / \mathrm{ml}$. Furthermore, the sensitivity, specificity, positive predictive value and negative predictive value of TFPI 2 for diagnosing VTE were 64.0, 80.4, 59.3 and 83.3\%, respectively. Additionally, $80.4 \%$ of patients with TFPI 2 levels $<398.9 \mathrm{pg} / \mathrm{ml}$ were VTE-negative. ROC analysis demonstrated that the area under the curve for TFPI2 was 0.729 (95\% confidence interval, 0.614-0.844). Conclusively, TFPI2 may distinguish patients with VTE from those without VTE among patients with EOC and positive D-dimer results.
\end{abstract}

Correspondence to: Dr Hiroshi Kobayashi, Department of Obstetrics and Gynecology, Nara Medical University, 840 Shijo-cho, Kashihara, Nara 634-8522, Japan

E-mail: hirokoba@naramed-u.ac.jp

Key words: D-dimer, ovarian cancer, tissue factor pathway inhibitor 2, venous thromboembolism

\section{Introduction}

Patients with cancer have an increased risk of venous thromboembolism (VTE) that varies substantially depending on the cancer type (1). Clear cell carcinoma (CCC) of the ovary constitutes $25 \%$ of epithelial ovarian cancer (EOC), exhibiting a higher incidence of VTE than other cancers (1). D-dimer, a soluble fibrin-degradation product, is a valuable marker for diagnosing VTE (2). The D-dimer test is frequently positive for VTE and inflammatory diseases, cancer, elderly age, surgery, trauma, pregnancy, and postpartum (3). However, the D-dimer test showed high sensitivity and negative predictive value (NPV) but low specificity for detecting VTE events (4). Imaging techniques, including venous ultrasound, computed tomography angiography, or both, are needed for diagnosing or excluding VTE (5). Thus, novel and effective strategies are needed to improve the specificity of VTE diagnosis and increase the clinical usefulness of the D-dimer test.

Tissue factor (TF) initiates the extrinsic coagulation pathway by binding to factor VIIa (FVIIa) and factor Xa (FXa) (the extrinsic coagulation initiation complex: TF-FVIIa-FXa) and plays a vital role in thrombosis development (6). Tissue factor pathway inhibitor (TFPI) is composed primarily of three Kunitz-type proteinase inhibitor domains (K1, K2, and K3) that downregulate TF-induced coagulation cascade (7). Furthermore, TFPI is classified into TFPI1 and TFPI2 [also known as placental protein 5 (PP5)] (7). Several studies have demonstrated that TFPI1 downregulation is a valuable clinical predictor of deep vein thrombosis (DVT) (8-13). Recently, TFPI2 has been verified as a novel serum marker for diagnosing EOC, especially CCC (14-16). However, no study has focused on TFPI2 as a marker for clinically diagnosing VTE in various cancers, including EOC. Therefore, this study assesses whether the TFPI2 test helps diagnose VTE in patients with EOC and positive D-dimer results.

\section{Materials and methods}

Patient population. From January 2008 to December 2015, 223 patients with EOC were treated at the Department of 
Gynecology, Nara Medical University Hospital. The patients were invited to give biobanking consent for future research. Informed consent for secondary use of biospecimens was obtained in August 2021 by an opt-out approach in all included patients. This study was approved by the Institutional Review Board and the Research and Ethical Committee of Nara Medical University Graduate School of Medicine Kashihara, Japan (no. 3061). Furthermore, plasma D-dimer levels were measured before surgery or chemotherapy. However, serum TFPI2 levels were measured using frozen serum samples from a biobank. Inclusion criteria were: i) patients undergoing surgery involving lesion removal for histological evaluation; ii) patients with histologically proven primary EOC; and iii) patients who could measure serum TFPI2 level using frozen pretreatment samples. The exclusion criteria were: i) age below 20 years; ii) a history of other malignancies or ovarian cancers other than EOC; iii) patients with no residual sample; iv) patients with negative D-dimer results (D-dimer $<1.0 \mathrm{ug} / \mathrm{ml}$ ); v) previous VTE; vi) currently suspected or symptomatic DVT or pulmonary thromboembolism (PTE); vii) lack of preoperative VTE screening (contraindications to iodinated contrast medium or emergency surgical intervention); viii) taking any drug affecting blood coagulation or fibrinolytic parameters (e.g., oral contraceptives, anticoagulant, antiplatelet drug, and fibrinolytic drugs); ix) severe concomitant comorbidities; and x) incomplete, inadequate, or missing data. In addition, the following clinicopathological, hematological, and imaging data were obtained from the patients' medical records: age, body mass index $\left(\mathrm{BMI} ; \mathrm{kg} / \mathrm{m}^{2}\right)$, serum TFPI2 level (pg/ml), plasma D-dimer level $(\mu \mathrm{g} / \mathrm{ml})$, serum CA125 level (U/ml), Federation of Gynecology and Obstetrics (FIGO) (International Federation of Gynecologists and Obstetricians) stage, histology, presence or absence of residual tumor, estimated ascites volume, history of VTE, anticoagulant use, and the presence or absence of comorbidities. Finally, stage classification was based on the FIGO 2014 guidelines.

Screening and detection of VTE. Primary VTE screening was performed by evaluating pathognomonic clinical signs or symptoms and plasma D-dimer levels. Clinical VTE features were painful leg swelling and tenderness, tachycardia, dyspnea, shortness of breath, chest pain, acute cardiovascular dysfunction, and loss of consciousness. Patients with clinical signs of VTE immediately underwent multidetector-row computed tomography scanning (SOMATOM Definition Flash, SOMATOM Definition As, and SOMATOM Emotion; Siemens Healthcare Diagnostics K.K.) with intravenously injected low-osmolar iodinated contrast mediums (17). All asymptomatic patients were initially evaluated by the D-dimer test on admission. Patients with positive D-dimer results $(>1.0 \mu \mathrm{g} / \mathrm{ml})$ further underwent bilateral whole-leg compression ultrasonography and contrast-enhanced CT chest, abdomen, and lower extremity scan to detect VTE (17). Furthermore, VTE was detected using a LOGIQ 9 ultrasound scanner (GE Healthcare) equipped with a 4-7 MHz transducer (17). Furthermore, contrast-enhanced CT scans were performed from the chest to the pelvis in patients with negative D-dimer results as a preoperative examination. Board-certified radiologists diagnosed VTE based on the image findings.
Collection and preservation of blood samples. Patients' peripheral venous blood samples were collected $\sim 4$ weeks before surgery or chemotherapy. First, $7 \mathrm{ml}$ venous blood samples were collected in vacuum tubes containing and not containing sodium citrate $(3.8 \%)$ from the cubital veins of subjects. Then, these tubes were centrifuged at 3,000 $\mathrm{x} g$ for at least $10 \mathrm{~min}$ at $4^{\circ} \mathrm{C}$. Finally, the separated sample was divided into $1,000 \mu \mathrm{l}$ aliquots and stored at $-80^{\circ} \mathrm{C}$ until further use.

Detection of plasma D-dimer and serum TFPI2. First, D-dimer concentrations were measured at the Department of Clinical Laboratory in Nara Medical University Hospital. Then, plasma D-dimer levels were measured using DD immunoenzymometric assay kit (Nanopia) using an automated immunoassay analyzer. Furthermore, VTE screening was performed based on the D-dimer levels, with a $1.0 \mu \mathrm{g} / \mathrm{ml}$ cutoff value for plasma D-dimer. Next, TFPI2 concentrations were measured at the Tosoh diagnostics product divisions (Tosoh Corporation, Kanagawa, Japan). Additionally, using two anti-TFPI2 monoclonal antibodies, the TFPI 2 concentration in serum samples was measured using E-test Tosoh II (AIA-PACK TFPI2) with an automated immunoassay analyzer ${ }^{\text {AIA }}-2000$ (Tosoh Corporation) (9). These assays demonstrated high reliability for intra-assay (2.2\%-2.7\%) and inter-assay (2.4\%-3.1\%) variability. Finally, measurements were performed by clinical laboratory technologists who were blinded to the study.

Statistical analysis. Statistical analysis was conducted using SPSS (Statistical Package for the Social Sciences, v.25, SPSS, Inc.). Clinicopathological characteristics were compared using the chi-squared test or Mann-Whitney $U$ test. The receiver-operating characteristic (ROC) curve was constructed, and areas under the curve were calculated to illustrate the diagnostic power of TFPI2. The maximum Youden index determined the best cutoff value for TFPI2. Furthermore, Spearman's correlation coefficient was used to analyze the correlation between D-dimer and TFPI 2 levels. Finally, $\mathrm{P}<0.05$ was considered to indicate a statistically significant difference.

\section{Results}

Characteristics of the study population and clinical features. A total of 223 subjects were admitted to our hospital during the research period. All patients had consented to biobanking, but 79 patients had no residual serum samples. However, 63 subjects were excluded from the study, including those with suspicious or symptomatic VTE $(n=2)$, those with negative D-dimer results $(<1.0 \mu \mathrm{g} / \mathrm{ml}, \mathrm{n}=41)$, those whose $\mathrm{D}$-dimer level was not measured $(n=12)$, and those with incomplete data $(n=8)$. Finally, 81 patients with EOC who met the inclusion and exclusion criteria participated in this study. VTE was objectively detected in 25 patients $(30.9 \%)$ and absent in 56 patients (69.1\%), and participant selection is shown in Fig. 1. Baseline characteristics of patients with EOC and with and without VTE are presented in Table I. Additionally, the median age was 60 years (range, 37-83 years), and CCC accounted for $22.2 \%$ of all EOC. During diagnosis, $66.7 \%$ of patients had advanced EOC. However, compared with the non-VTE group, the VTE group had higher D-dimer and TFPI2 levels $(\mathrm{P}=0.004$ and $\mathrm{P}=0.001$, respectively). In contrast, there were 
Table I. Clinicopathological characteristics of patients with epithelial ovarian cancer, with and without VTE.

\begin{tabular}{|c|c|c|c|}
\hline Factor & $\operatorname{VTE}(n=25)$ & non-VTE $(n=56)$ & P-value \\
\hline Age, year, median (range) & $58(40-81)$ & $61(37-83)$ & $0.546^{\mathrm{a}}$ \\
\hline BMI, $\mathrm{kg} / \mathrm{m}^{2}$, median (range) & $22.6(17.0-36.0)$ & $22.0(15.2-34.3)$ & $0.101^{\mathrm{a}}$ \\
\hline TFPI2, pg/ml, median (range) & $472.2(199.4-1,288.4)$ & $279.1(116.1-5,824.2)$ & $0.001^{\mathrm{a}}$ \\
\hline D-dimer, $\mu \mathrm{g} / \mathrm{ml}$, median (range) & $5.1(1.2-35.2)$ & $2.6(1.1-15.5)$ & $0.004^{\mathrm{a}}$ \\
\hline CA125, U/ml, median (range) & $1,021.6(17.5-8,386.0)$ & $503.3(8.2-43,170.9)$ & $0.236^{\mathrm{a}}$ \\
\hline \multicolumn{4}{|l|}{ Massive ascites ${ }^{\mathrm{c}}, \mathrm{n}$} \\
\hline No & 13 & 31 & \multirow[t]{2}{*}{$0.779^{\mathrm{b}}$} \\
\hline Yes & 12 & 25 & \\
\hline \multicolumn{4}{|l|}{ Residual tumor, $\mathrm{n}$} \\
\hline No & 8 & 21 & \multirow[t]{2}{*}{$0.663^{\mathrm{b}}$} \\
\hline Yes & 17 & 35 & \\
\hline \multicolumn{4}{|l|}{ Histology, n } \\
\hline $\mathrm{CCC}$ & 6 & 12 & \multirow[t]{2}{*}{$0.797^{\mathrm{b}}$} \\
\hline Non-CCC & 19 & 44 & \\
\hline \multicolumn{4}{|l|}{ FIGO stage $^{\mathrm{d}}, \mathrm{n}$} \\
\hline $\mathrm{I} / \mathrm{II}$ & 9 & 18 & \multirow[t]{2}{*}{$0.734^{\mathrm{b}}$} \\
\hline III/IV & 16 & 38 & \\
\hline
\end{tabular}

a, Mann-Whitney $U$ test; ${ }^{\text {b }}$, Chi-squared test; ${ }^{\text {c }}$, as assessed by computed tomography, massive ascites were defined as ascitic fluid beyond the small pelvic cavity; ${ }^{\mathrm{d}}, 2014$. TFPI2, tissue factor pathway inhibitor 2; VTE, venous thromboembolism; BMI, body mass index; CCC, clear cell carcinoma of the ovary; FIGO, International Federation of Gynecology and Obstetrics.

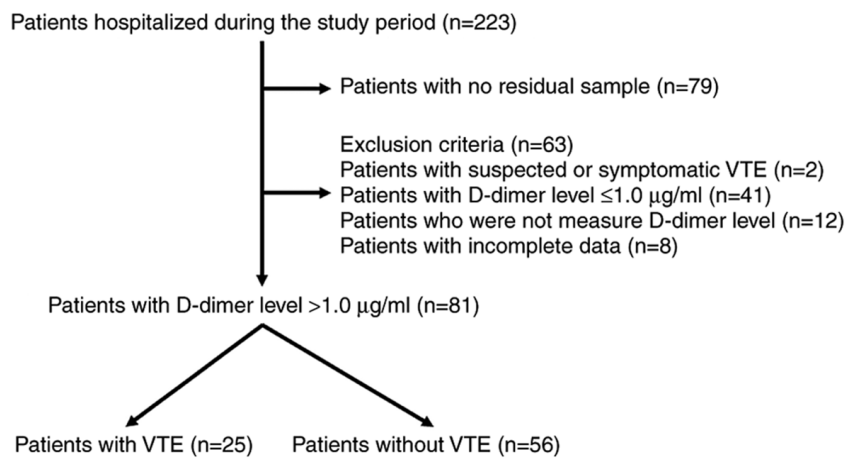

Figure 1. Selection of participants. Of the 223 women who met the inclusion criteria, 63 were excluded as they met the exclusion criteria, while 81 patients participated in the study. Of these, 25 women had VTE, and 56 did not. TFPI2, tissue factor pathway inhibitor 2; VTE, venous thromboembolism.

no differences between both groups in age, BMI, CA125 level, ascites volume, residual tumor, histology, and FIGO stage.

Serum TFPI2 level in patients with VTE and non-VTE. We examined serum TFPI2 distribution in patients with non-VTE $(n=56)$ and VTE $(n=25)$ by Box and Whisker plot analysis. Also, serum TFPI2 levels in patients with VTE are significantly higher than those with non-VTE (Mann-Whitney $U$ test, $\mathrm{P}=0.001$; Fig. 2).

TFPI2 diagnostic value. ROC curve analysis was performed to assess the TFPI2 level between VTE and non-VTE groups in patients with EOC and positive D-dimer results (Fig. 3). The area under the ROC curve was 0.729 [95\% confidence interval

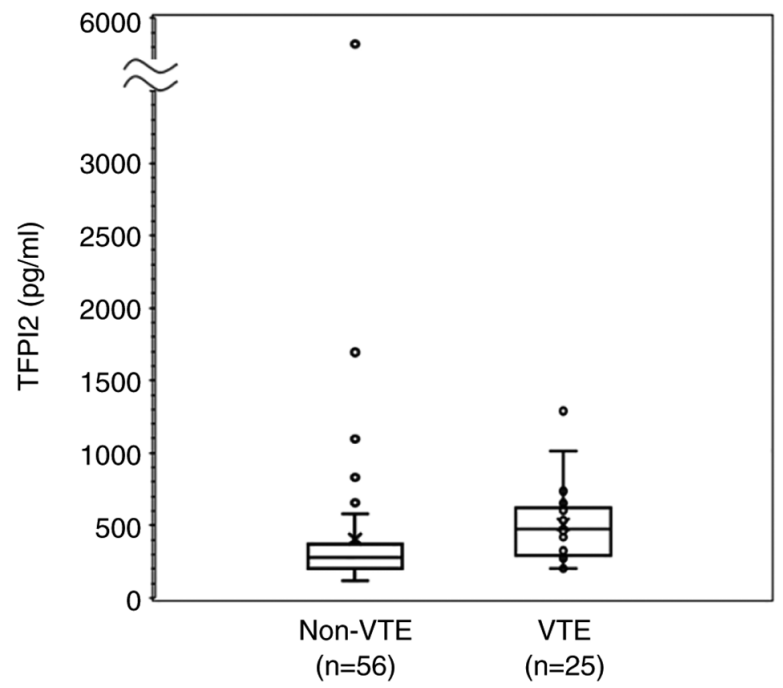

Figure 2. Serum TFPI2 level in patients with VTE and non-VTE. Box and whisker plot analysis showing the distribution of TFPI2 values for VTE $(n=25)$ and non-VTE patients $(n=56)$. The median TFPI2 level was $472.2 \mathrm{pg} / \mathrm{ml}$ (IQR, 329.5) in patients with VTE and $279.1 \mathrm{pg} / \mathrm{ml}$ (IQR, 164.1) in patients without VTE. The difference in TFPI2 levels between these groups was statistically significant (Mann-Whitney $\mathrm{U}$ test, $\mathrm{P}=0.001$ ). TFPI2, tissue factor pathway inhibitor 2; VTE, venous thromboembolism; $\mathrm{IQR}$, interquartile range.

(CI) 0.614-0.844]. Using the Youden index, the optimal cutoff value for the TFPI2 level was set at $398.9 \mathrm{pg} / \mathrm{ml}$. With this cutoff value, the sensitivity, specificity, PPV, and NPV of the TFPI2 test were $64.0,80.4,59.3$, and $83.3 \%$, respectively, in diagnosing VTE. 


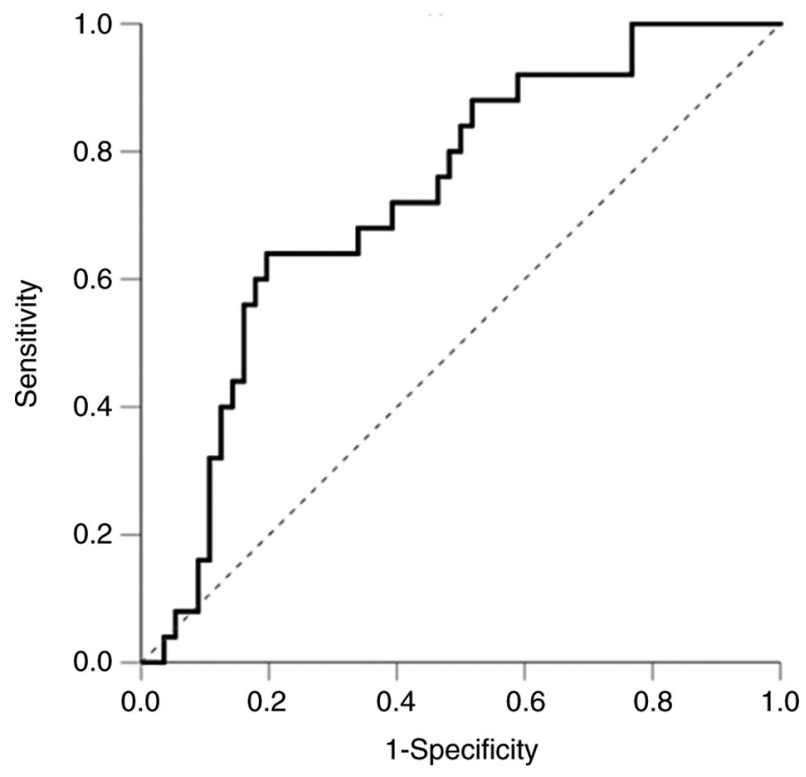

Figure 3. Receiver-operating curve analysis of TFPI2 levels in distinguishing between VTE and non-VTE groups in patients with epithelial ovarian cancer and positive D-dimer results. TFPI2, tissue factor pathway inhibitor 2; VTE, venous thromboembolism.

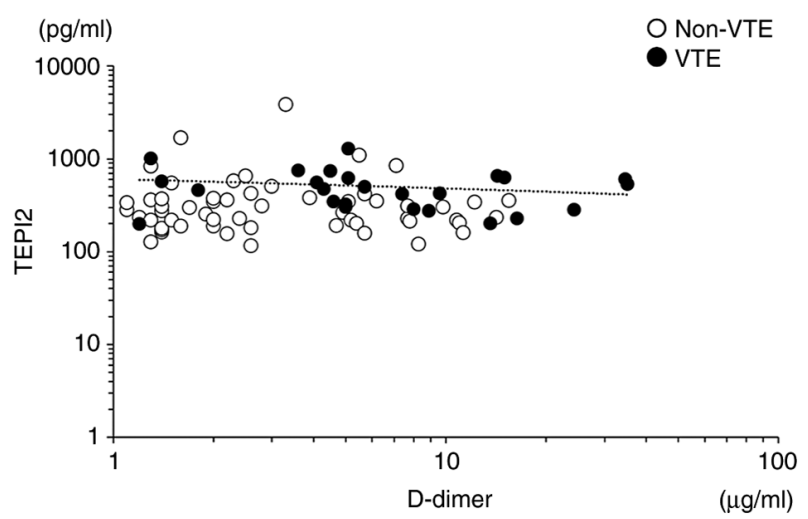

Figure 4. Correlation between D-dimer and TFPI2 levels. Spearman's correlation coefficient was used to examine the association between the two markers. TFPI2, tissue factor pathway inhibitor 2; VTE, venous thromboembolism.

Correlations between D-dimer and TFPI2 levels. The correlation between D-dimer and TFPI2 levels is presented in Fig. 4. The Spearman correlation coefficient between the levels of $\mathrm{D}$-dimer and TFPI2 was 0.063 ( $\mathrm{P}=0.574)$. Finally, there was no significant correlation between D-dimer and TFPI2 levels.

\section{Discussion}

TFPI2 is a novel serodiagnostic marker for EOC (14-16). TFPI2 levels were significantly increased in patients with CCC than those without CCC, suggesting that TFPI2 may help diagnose CCC (14-16). The TFPI2 test has been covered by the Japanese national health insurance system since April 2021. Furthermore, we showed for the first time that TFPI2 might effectively diagnose asymptomatic VTE in patients with EOC and positive D-dimer assay results. Additionally, in patients with EOC and positive D-dimer results, the sensitivity and specificity of the
TFPI2 test were 64.0 and $80.4 \%$, respectively, in diagnosing VTE, with a cutoff value of $398.9 \mathrm{pg} / \mathrm{ml}$. Finally, $80.4 \%$ of patients with TFPI 2 levels $<398.9 \mathrm{pg} / \mathrm{ml}$ were VTE negative.

First, EOC, especially CCC, increases the risk of VTE $(1,18)$. DVT was detected in $22.7 \%$ of patients with EOC, which was higher than those $(<10 \%)$ reported in other malignancies (19). Additionally, women with CCC were associated with a 2.5 times higher risk for VTE than women with other histological types of EOC (18). More than $80 \%$ of women with DVT were asymptomatic, and accurately predicting VTE before treatment is challenging (18). Therefore, a reliable, non-invasive diagnostic and screening tool for accurately evaluating VTE is needed. D-dimer testing is the most widely used for VTE evaluation, but its low specificity requires definitive confirmation by imaging techniques (20). Venous ultrasonography and contrast-enhanced CT scans are expensive and time-consuming, so novel biomarkers that enhance the diagnostic performance combined with D-dimer are required. Furthermore, TFPI2 may be a serodiagnostic marker that fits this purpose. It is unclear whether two-step screening with D-dimer and TFPI2 is clinically more beneficial than D-dimer and ultrasonography, but it may reduce the burden on the ultrasound specialist. However, we cannot compare the diagnostic performance of TFPI2 with that of D-dimer because the former was not measured in all patients. In addition, the sensitivity and specificity of TFPI 2 are targeted for patients with a D-dimer $>1 \mu \mathrm{g} / \mathrm{ml}$.

Second, TFPI, including TFPI1 and TFPI2, mainly inhibits blood coagulation induced by TF. TF, a potent initiator of the extrinsic coagulation cascade, can be released into the systemic circulation from viable tumor cells, especially CCC, activating the coagulation system and increasing the risk of DVT (21). TFPI1 levels were significantly downregulated in patients with DVT compared with non-DVT patients (8-13), suggesting that low TFPI1 levels increase DVT risk. Surprisingly, TFPI2 levels were increased in patients with VTE compared with TFPI1 levels. Elevated TFPI2 should suppress TF activity, inhibiting thrombus formation. Rather, elevated TFPI2 is a compensatory mechanism that may reflect beneficial adaptations to maintain blood coagulation homeostasis. In contrast, TFPI2, unlike TFPI1, acts as a potent plasmin inhibitor. Plasmin inactivates coagulation factors, FV, FVIII, FIX, and FX (22). Plasmin is also involved in fibrinolysis and contributes to fibrin degradation and clot removal (23). TFPI2 may inhibit the removal of organized clots by plasmin. Elucidation of the mechanism of TFPI 2 production and thrombosis formation in EOC is awaited.

Finally, there are several limitations to this study. First, this is a single-center study conducted in the Department of Gynecology at University Hospital. Second, This study is limited to the diagnosis of asymptomatic DVT in patients with positive D-dimer results, hampering the broader applicability in clinical practice. Third, there is currently no evidence that TFPI2 has higher specificity than D-dimer. Further study is needed to assess whether TFPI2 provides the clinical utility with the current D-dimer test to improve VTE diagnosis.

Conclusively, TFPI2 may improve the diagnostic performance of asymptomatic VTE in patients with EOC and positive D-dimer results. However, a prospective study using a larger cohort of patients with EOC is needed to determine 
if the combination test of the two markers helps screen for detecting VTE.

\section{Acknowledgements}

Not applicable.

\section{Funding}

This study was supported by the Japan Society for the Promotion of Science (JSPS) (grant no. 20K09647).

\section{Availability of data and materials}

The datasets used and/or analyzed during the current study are available from the corresponding author on reasonable request.

\section{Authors' contributions}

HK made substantial contributions to conception and design. RM, SY and RK were responsible for the acquisition of data. NO and SM contributed to measuring TFPI2 levels. YY was responsible for the analysis and interpretation of data. RM and YY confirm the authenticity of all the raw data. The first draft of the manuscript was written by RM under the guidance of HK. All authors have read and approved the final manuscript.

\section{Ethics approval and consent to participate}

The present study was approved by the medical ethics committee of the Nara Medical University (approval no. 3061; Kashihara, Japan). Written informed consent was obtained from each patient. All procedures followed were in accordance with the ethical standards of the responsible committee on human experimentation (institutional and national) and with the Helsinki Declaration of 1964 and its later amendments.

\section{Patient consent for publication}

Not applicable.

\section{Competing interests}

The authors declare that they have no competing interests.

\section{References}

1. Matsuura Y, Robertson G, Marsden DE, Kim SN, Gebski V and Hacker NF: Thromboembolic complications in patients with clear cell carcinoma of the ovary. Gynecol Oncol 104: 406-410, 2007.

2. Weitz JI, Fredenburgh JC and Eikelboom JW: A test in context: D-dimer. J Am Coll Cardiol 70: 2411-2420, 2017.

3. Hou H, Ge Z, Ying P, Dai J, Shi D, Xu Z, Chen D and Jiang Q: Biomarkers of deep venous thrombosis. J Thromb Thrombolysis 34: 335-346, 2012.

4. Wells PS, Anderson DR, Rodger M, Forgie M, Kearon C, Dreyer J, Kovacs G, Mitchell M, Lewandowski B and Kovacs MJ: Evaluation of D-dimer in the diagnosis of suspected deep-vein thrombosis. N Engl J Med 349: 1227-1235, 2003.
5. Andrews EJ Jr and Fleischer AC: Sonography for deep venous thrombosis: Current and future applications. Ultrasound Q 21: 213-225, 2005.

6. Kocatürk B and Versteeg HH: Tissue factor-integrin interactions in cancer and thrombosis: Every Jack has his Jill. J Thromb Haemost 11 (Suppl 1): S285-S293, 2013.

7. Peraramelli S, Thomassen S, Heinzmann A, Rosing J, Hackeng TM, Hartmann R, Scheiflinger F and Dockal M: Inhibition of tissue factor:factor VIIa-catalyzed factor IX and factor $\mathrm{X}$ activation by TFPI and TFPI constructs. J Thromb Haemost 12: 1826-1837, 2014.

8. Fei X, Wang H, Yuan W, Wo M and Jiang L: Tissue factor pathway inhibitor-1 is a valuable marker for the prediction of deep venous thrombosis and tumor metastasis in patients with lung cancer. Biomed Res Int 2017: 8983763, 2017.

9. Sidelmann JJ, Bladbjerg EM, Gram J, Münster AM and Jespersen J: Tissue factor pathway inhibitor relates to fibrin degradation in patients with acute deep venous thrombosis. Blood Coagul Fibrinolysis 19: 405-409, 2008.

10. Dahm A, Rosendaal FR, Andersen TO and Sandset PM: Tissue factor pathway inhibitor anticoagulant activity: Risk for venous thrombosis and effect of hormonal state. Br J Haematol 132: 333-338, 2006.

11. Dahm A, Van Hylckama Vlieg A, Bendz B, Rosendaal F, Bertina RM and Sandset PM: Low levels of tissue factor pathway inhibitor (TFPI) increase the risk of venous thrombosis. Blood 101: 4387-4392, 2003.

12. Amini-Nekoo A, Futers TS, Moia M, Mannucci PM, Grant PJ and Ariëns RA: Analysis of the tissue factor pathway inhibitor gene and antigen levels in relation to venous thrombosis. Br J Haematol 113: 537-543, 2001.

13. Fedi S, Gori AM, Falciani M, Cellai AP, Aglietti P, Baldini A, Vena LM, Prisco D, Abbate R and Gensini GF: Procedure-dependence and tissue factor-independence of hypercoagulability during orthopaedic surgery. Thromb Haemost 81: 874-878, 1999.

14. Arakawa N, Miyagi E, Nomura A, Morita E, Ino Y, Ohtake N, Miyagi Y, Hirahara F and Hirano H: Secretome-based identification of TFPI2, a novel serum biomarker for detection of ovarian clear cell adenocarcinoma. J Proteome Res 12: 4340-4350, 2013

15. Arakawa N, Kobayashi H, Yonemoto N, Masuishi Y, Ino Y, Shigetomi H, Furukawa N, Ohtake N, Miyagi Y, Hirahara F, et al: Clinical significance of tissue factor pathway inhibitor 2, a serum biomarker candidate for ovarian clear cell carcinoma. PLoS One 11: e0165609, 2016

16. Miyagi E, Arakawa N, Sakamaki K, Yokota NR, Yamanaka T, Yamada Y, Yamaguchi S, Nagao S, Hirashima Y, Kasamatsu Y, et al: Validation of tissue factor pathway inhibitor 2 as a specific biomarker for preoperative prediction of clear cell carcinoma of the ovary. Int J Clin Oncol 26: 1336-1344, 2021.

17. Kawaguchi R, Furukawa $\mathrm{N}$ and Kobayashi H: Cut-off value of D-dimer for prediction of deep venous thrombosis before treatment in ovarian cancer. J Gynecol Oncol 23: 98-102, 2012.

18. Duska LR, Garrett L, Henretta M, Ferriss JS, Lee L and Horowitz N: When 'never-events' occur despite adherence to clinical guidelines: The case of venous thromboembolism in clear cell cancer of the ovary compared with other epithelial histologic subtypes. Gynecol Oncol 116: 374-377, 2010.

19. Ebina Y, Uchiyama M, Imafuku H, Suzuki K, Miyahara Y and Yamada H: Risk factors for deep venous thrombosis in women with ovarian cancer. Medicine (Baltimore) 97: e11009, 2018.

20. Bates SM, Jaeschke R, Stevens SM, Goodacre S, Wells PS, Stevenson MD, Kearon C, Schunemann HJ, Crowther M, Pauker SG, et al: Diagnosis of DVT: Antithrombotic therapy and prevention of thrombosis, 9th ed: American college of chest physicians evidence-based clinical practice guidelines. Chest 141 (Suppl 2): e351S-e418S, 2012.

21. Geddings JE and Mackman N: Tumor-derived tissue factorpositive microparticles and venous thrombosis in cancer patients. Blood 122: 1873-1880, 2013.

22. Hoover-Plow J: Does plasmin have anticoagulant activity? Vasc Health Risk Manag 6: 199-205, 2010.

23. Medcalf RL: Fibrinolysis: from blood to the brain. J Thromb Haemost 15: 2089-2098, 2017. 